Annuaire du Collège de France 2017-2018

\title{
Hyperkähler varieties
}

\section{Kieran Gregory O'Grady}

\section{(2) OpenEdition}

\section{Journals}

Electronic version

URL: https://journals.openedition.org/annuaire-cdf/16427

DOI: 10.4000/annuaire-cdf.16427

ISBN: 978-2-7226-0572-5

ISSN: 2109-9227

Publisher

Collège de France

Printed version

Date of publication: 30 December 2020

Number of pages: $704-705$

ISBN: 978-2-7226-0516-9

ISSN: 0069-5580

\section{Electronic reference}

Kieran Gregory O'Grady, "Hyperkähler varieties", L'annuaire du Collège de France [Online], 118 | 2020,

Online since 01 April 2021, connection on 22 August 2022. URL: http://journals.openedition.org/ annuaire-cdf/16427 ; DOI: https://doi.org/10.4000/annuaire-cdf.16427

All rights reserved 


\title{
HYPERKÄHLER VARIETIES
}

\author{
Kieran Gregory O'GRADY
}

Professeur à l'université de la Sapienza de Rome (Italie)

Mots-clés : hyperkähler varieties

Cycle de trois leçons données les 14, 15 et 22 février 2018 (invitation sur proposition du Pr Claire Voisin), disponibles en audio sur le site internet du Collège de France : https://www.college-de-france.fr/site/claire-voisin/guestlecturer-2017-2018.htm.

\section{HYPERKÄHLER VARIETIES I}

A hyperkähler (HK) manifold is a compact simply connected Kähler manifold carrying a holomorphic symplectic form spanning the space of holomorphic 2 forms.

The Beauville Bogomolov decomposition Theorem (based on key results of De Rham, Berger, Yau...) highlights the relevance of HK manifolds, because it states that a compact Kähler manifold with torsion first Chern class is, up to a finite étale cover, a product of Kähler manifolds of the following types: a compact complex torus, a Calabi-Yau variety (i.e. simply connected, with trivial canonical bundle and no non non zero holomorphic form of intermediate degree, of dimension at least 3 ), and a HK manifold.

The first lecture was a gentle introduction to the theory of HK manifolds.

A HK manifold of dimension 2 is a $K 3$ surface. I described Beauville's examples of hyperkähler manifolds in arbitrary even dimension, i.e. Hilbert schemes of finite length subschemes of a $K 3$ surface, and generalized Kummers associated to 2 dimensional complex tori. I went through the basic (soft) results on deformations and on the Beauville-Bogomolov-Fujiki quadratic form. I demonstrated the power of the latter by sketching the proof of Matsushita's Theorem on Lagrangian fibrations. I mentioned (hard) fundamental results, such as existence of twistor families, Huybrechts' (and Boucksom's) description of the Kähler cone, and Verbitsky's global Torelli Theorem.

\section{HYPERKÄHLER VARIETIES II}

A construction of Kuga and Satake associates to a HK manifold $X$ a compact complex torus $K S(X)$ and an embedding of the second cohomology of $X$ into the second cohomology of the product of $K S(X)$ with its dual (respecting the Hodge structures). If the HK manifold is polarized (hence projective), then $K S(X)$ is polarized as well. The definition of $K S(X)$ is purely transcendental, but the Hodge conjecture predicts that if $X$ is projective, then the embedding of Hodge structures mentioned above is realized by an algebraic cycle. Very few examples are known for 
which there is a geometric description of the Kuga Satake torus, and a verification that the Kuga Satake cycle class is algebraic.

I presented my results on the Kuga Satake torus of a HK manifold which is a deformation of a generalized Kummer. If $X$ is such a HK manifold, then its third Betti number is 8 , hence there is an associated intermediate Jacobian $J(X)$, a compact complex torus of dimension 4. My results can be summarized as stating that $J(X)$ is as good as the Kuga Satake torus if one wants to realize the second cohomology of $X$ as a Hodge substructure of the second cohomology of a torus. On the other hand, $J(X)$ has some clear advantages when compared to $K S(X)$ : it is of smallerdimension, and it is geometrically closer to $X$ because of the Abel-Jacobi map.

\section{HYPERKÄHLER VARIETIES III}

Moduli spaces of polarized HK varieties exist and are quasi projective varieties - this is a particular instance of fundamental results of Viehweg. Unless one is in a hypothetical case of HK's with second Betti number equal to 3, the moduli space is not projective. I presented an ongoing project of Radu Laza and myself which deals with projective birational models of the moduli spaces (in certain cases).

The starting point is Verbitsky's Global Torelli Theorem, which identifies the moduli space with a locally symmetric quasi projective variety (of Type IV). Hence the moduli space can be completed to the projective Baily Borel compactification, which is minimal, explicit, but a priori not a modular compactification. In certain instances, e.g. $K 3$ surfaces of low degree, the moduli space has a birational model which is a GIT moduli space which has a fairly explicit description. Looijenga defined new projective birational models of the relevant locally symmetric spaces in order to give an explicit "arithmetic" description of the GIT moduli space. The work of Laza and myself aims to pursue and make more precise Looijenga's program, and is inspired also by the Hassett-Keel program on canonical models of the moduli space of curves. 